\title{
Transtorno bipolar
}

Martin Alda ${ }^{1}$

\section{Resumo}

Os resultados de estudos de famílias sugerem que o transtorno bipolar tenha uma base genética. Essa hipótese foi reforçada em estudos de adoção e de gêmeos. A herança do transtorno bipolar é complexa, envolve vários genes, além de apresentar heterogeneidade e interação entre fatores genéticos e não-genéticos. Achados, que já foram replicados, já implicaram os cromossomos 4, 12, 18 e 21, entre outros, na busca por genes de suscetibilidade. Os resultados mais promissores foram obtidos através de estudos de ligação. Por outro lado, os estudos de associação geraram dados interessantes, mas ainda vagos. Os estudos de populações de pacientes homogêneos e a melhor definição do fenótipo deverão contribuir para avanços futuros. A identificação dos genes relacionados ao transtorno bipolar irá permitir o melhor entendimento e tratamento dessa doença.

\section{Descritores}

Transtorno bipolar; genética; ligação; associação

\section{Abstract}

Family studies of bipolar disorder have suggested that the illness might have a genetic basis. This hypothesis has been also supported by twin and adoption studies. The inheritance of bipolar disorder follows a complex pattern likely including a contribution of several genes, heterogeneity, and interaction of genetic and non-genetic factors. The search for specific susceptibility genes has now generated replicated findings on chromosomes 4,12, 18 and 21, and possibly several others. The most promising results have been obtained using the linkage strategy, while the association studies have generated perhaps stimulating, but less definitive findings. Further progress may benefit from studies of homogeneous patient populations and an improved phenotype definition. Identification of bipolar-disorder genes will contribute to better understanding and treatment of this illness.

\section{Keywords}

Bipolar disorder; genetics; linkage; association

\section{Introdução}

O transtorno bipolar (TBP) é uma condição psiquiátrica relativamente freqüente, com prevalência na população entre $1 \%$ e $2 \%$. É caracterizado por episódios de alteração do humor de difícil controle - depressão ou mania (bipolar I) ou depressão e hipomania (bipolar II). Os sintomas podem aparecer em qualquer idade, sendo mais comum o surgimento entre o início da segunda e meio da terceira década de vida. A etiologia da doença ainda não é conhecida, mas muitos estudos apontam para a existência de disfunções complexas, incluindo alterações nos receptores e nos pós-receptores de neurotransmissores.

\section{Inato ou adquirido?}

Alguns dos primeiros dados sobre a agregação familiar do transtorno bipolar provêm de estudos conduzidos no final dos anos 50. Esses estudos determinaram a prevalência do TBP e de outras doenças psiquiátricas entre parentes de probandos com TBP e, em alguns casos, entre parentes de indivíduoscontrole saudáveis. Como pode ser visto na tabela 1, há uma grande variabilidade de riscos, de acordo com os critérios diagnósticos utilizados.

Entretanto, quando analisados conjuntamente, esses estudos encontraram um risco 8 a 15 vezes maior em relação à prevalência na população em geral. Os estudos de família, isoladamente, não provam a natureza genética (ao invés da familiar) da doença. Já os estudos de adoção e de gêmeos têm maior poder de distinção entre os componentes genéticos e ambientais. No caso do TBP, esses estudos fornecem evidências de uma natureza genética para a doença (Gershon et a ${ }^{18}$ or Alda ${ }^{19}$ para revisões).

\section{O que é herdado?}

Além de sugerirem a presença de um componente genético para o TBP, os estudos de famílias também indicam a possibilidade de outras condições psiquiátricas fazerem parte de um mesmo espectro fenotípico. Em outras palavras, essas condições podem ser todas manifestações diversas de uma mesma predisposição genética. A depressão unipolar é encontrada mais 
Tabela 1 - Riscos de morbidade (\%) de distúrbio bipolar e de depressão unipolar em parentes de primeiro grau de probandos com DBP.

\begin{tabular}{|c|c|c|}
\hline Estudo & Distúrbio bipolar & Depressão unipolar \\
\hline Stenstedt ${ }^{1}$ & 5.0 & 8.0 \\
\hline Perris $^{2}$ & 10.2 & 10.5 \\
\hline Angst $^{3}$ & 3.7 & 11.2 \\
\hline Goetzl et al. ${ }^{4}$ & 4.0 & 21.0 \\
\hline Helzer e Winokur ${ }^{5}$ & 5.0 & 12.0 \\
\hline Gershon et al. ${ }^{6}$ & 3.8 & 6.8 \\
\hline Videbech $^{7}$ & 8.0 & 6.0 \\
\hline James e Chapman ${ }^{8}$ & 6.4 & 13.2 \\
\hline Angst et al. ${ }^{9}$ & 5.6 & 6.2 \\
\hline Tsuang et al. ${ }^{10}$ & 3.9 & 9.1 \\
\hline Taylor et al. ${ }^{11}$ & 4.8 & 4.2 \\
\hline Gershon et al. ${ }^{12}$ & 8.0 & 14.9 \\
\hline Baron et al. ${ }^{13}$ & 4.5 & 16.3 \\
\hline Fieve et al. ${ }^{14}$ & 2.9 & 8.4 \\
\hline Coryell et al. ${ }^{15}$ & 7.0 & 22.4 \\
\hline Tsuang et al. ${ }^{16}$ & 3.9 & 9.1 \\
\hline Rice et al. ${ }^{17}$ & 10.6 & 24.3 \\
\hline
\end{tabular}

freqüentemente entre parentes de probandos com TBP do que em parentes de controles psiquiatricamente saudáveis. Outra condição que possivelmente faz parte do espectro bipolar é o transtorno esquizoafetivo, do tipo maníaco. Já em relação a outras entidades, como ciclotimia, transtornos de personalidade, de alimentação ou alcoolismo, os dados são mais ambíguos. Todas essas condições já foram, em vários estudos, implicadas como parte do espectro bipolar, mas as evidências são ainda tênues.

\section{Como a doença é transmitida de uma geração para a outra?}

Desde 1969, diversos grupos propõem que o TBP tenha uma herança dominante ligada ao X. As observações iniciais que geraram esta hipótese foram a ausência relativa de transmissão pai-filho e a presença de ligação com o daltonismo protan-deutan..$^{20-22}$ Esses resultados estimularam novos estudos: alguns refutam e outros sustentam a hipótese de herança ligada ao X. Resultados mais recentes sugerem que a região cromossômica Xq26 seja a portadora do suposto locus do TBP. ${ }^{23}$ No entanto, é improvável que a transmissão pelo cromossomo $\mathrm{X}$ ocorra em todas as famílias com TBP, ou mesmo em proporção significativa delas.

Conforme discutido por Hebebrand, ${ }^{24}$ essa transmissão implicaria que todas as filhas de homens afetados fossem também afetadas (ou ao menos uma grande proporção delas, devido à penetrância incompleta), o que não é visto em dados de prevalência em famílias. Resultados igualmente conflitantes vieram de estudos que testaram o modelo autossômico de herança. Os resultados dos estudos de segregação são mais bem compreendidos se interpretados como geradores de hipóteses e não como forma de verificação delas. Podem ajudar nas análises paramétricas de ligação, mas seu valor não deve ser superestimado. A opinião atual é que o TBP seja herdado como um traço oligogênico ou multifatorial. Também é possível que algumas famílias com a doença apresentem segrega- ção de um único gene - ou por ser o traço monogênico, ou porque apenas um entre os diversos genes envolvidos é polimórfico naquela família.

\section{A busca pelos genes de suscetibilidade \\ Estudos de ligação}

Os estudos de ligação genética do TBP já ocorrem há mais de três décadas. Os primeiros estudos promissores originaram-se de análises de ligação com o cromossomo X.21, 22, 25 Entretanto, houve uma série de problemas metodológicos com esses estudos, além de falha em reproduzir os achados relacionados à região cromossômica Xq28. ${ }^{26,27}$ Também malsucedidas foram as tentativas de reproduzir os resultados, inicialmente promissores, com os marcadores do cromossomo $11 \mathrm{p}$, região que contém o gene candidato da tirosina hidroxilase..$^{28,29}$

Na década passada, entretanto, diversas regiões atraíram atenção, tanto pelo poder de ligação relatado, como pelos resultados de vários estudos independentes.

Um dos cromossomos mais estudados foi o 18. Após o relato inicial por Berrettini et al, ${ }^{30}$ que sugeriu a presença de ligação com a região pericentromérica, diversos outros estudos confirmaram esse achado. ${ }^{31,32}$ Além disso, outros estudos sugeriram ligação com pelo menos duas outras regiões distintas no braço longo do cromossomo. ${ }^{31,33}$ Por outro lado, estudos metodologicamente corretos não encontraram a presença de ligação, sugerindo a existência de heterogeneidade genética. Outra região de ligação promissora, já reproduzida, é a região cromossômica 21q. ${ }^{37-39}$ Atualmente, uma das ligações encontradas mais poderosas localizase na região $12 q 23$-q24. ${ }^{40}$ Por último, o achado de ligação significativa com o cromossomo $4 \mathrm{p} 16^{41}$ foi confirmado em ao menos um estudo. ${ }^{42}$

Estudos adicionais também relataram outras ligações significativas, com resultados que, apesar de ainda não reproduzidos, parecem ser bastante promissores. Há, por exemplo, os resultados do rastreamento do genoma da amostra do NIMH, que sugerem a existência de diversos loci de suscetibilidade nos cromossomos $1,6,7,10,16$ e $22 ;{ }^{43}$ outro estudo recente sugere loci nas regiões $13 \mathrm{q} 32 \mathrm{e} 1 \mathrm{q} 32 .{ }^{32}$

Vale notar também os dados do estudo Amish, que avaliou a ligação entre um potencial fator de proteção e marcadores genéticos. Este estudo sugere a presença de um ou mais loci que aumentariam a probabilidade de não-acometimento entre parentes de probandos com TBP. Foram encontrados dois loci no cromossomo 4 , um na região 4 p e outro na região $3 q .{ }^{44}$

\section{Estudos de associação}

Os estudos de associação são freqüentemente vistos como complementares aos estudos de ligação. Maior interesse foi dado aos possíveis genes candidatos, isto é, genes que, de acordo com conhecimento prévio, estariam possivelmente relacionados ao aumento de suscetibilidade para TBP. Exemplos incluem os genes da tirosina hidroxilase, do transportador de serotonina, diversos genes receptores ou genes envolvidos na regulação da transdução de sinal. Até o presente momento, nenhum dado consistente e reproduzido emergiu desses estudos. 


\section{Repetições de trinucleotídeos e antecipação}

Diversos transtornos neurovegetativos, nos quais ocorre o fenômeno da antecipação (início mais precoce e formas mais graves da doença a cada nova geração), são causados pela expansão de seqüências de repetições de trinucleotídeos, sendo mais comum a repetição de CAG. Diversos estudos de esquizofrenia e TBP sugeriram a ocorrência de antecipação nessas duas condições, desencadeando, com isso, a busca por repetições instáveis de trinucleotídeos. ${ }^{45}$ Até o momento, os resultados são negativos ou duvidosos. Mesmo os estudos que demonstraram um aumento no comprimento de CAG, não encontraram nenhuma associação entre o tamanho da expansão e as características clínicas da doença, como idade de início dos sintomas. ${ }^{46}$

\section{Direções futuras}

O conhecimento sobre a biologia e a genética do TBP avançou muito. O TBP é uma das condições psiquiátricas na qual a identificação de genes parece ser mais provável. Ao mesmo tempo, os pesquisadores concordam que algumas melhorias metodológicas são necessárias para que tal objetivo seja atingido. Um dos pontos essenciais é definir adequadamente o fenótipo. O diagnóstico clínico, estabelecido através de critérios diagnósticos, é confiável, mas sua validade ainda não foi determinada. Um fenótipo mais próximo à condição genética da doença seria mais adequado para a análise genética.

Outro problema, também relacionado ao diagnóstico, é a provável heterogeneidade da doença. A heterogeneidade torna mais difícil a pesquisa por genes de suscetibilidade. Estudos de subgrupos homogêneos são vantajosos. Entretanto, subgrupos de TBP não são facilmente reconhecidos, nem bem estabelecidos. Uma abordagem promissora é classificar os participantes da pesquisa de acordo com a resposta ao tratamento - profilaxia com lítio, ${ }^{47}$ ou outras formas de tratamento, como com fluvoxamine na depressão maior. ${ }^{48}$ Acredita-se que as famílias de probandos com doença de início precoce tenham maior grau de "carga genética", ${ }^{49}$ sendo assim um subgrupo particularmente interessante para pesquisa. Outra forma de definição fenotípica seria combinar o diagnóstico clínico a marcadores biológicos. Alguns dos achados mais promissores dos estudos de ligação foram obtidos a partir de populações etnicamente homogêneas, como os franco-canadenses, ${ }^{40}$ a população $A m i s h^{44}$ ou costarriquenhos. ${ }^{33}$

Apesar de todas essas estratégias ainda precisarem provar seu valor na busca pela identificação de genes que predispõem ao TBP, tal descoberta certamente terá implicações importantes, tanto no entendimento, como no tratamento dessa grave condição.

\section{Referências bibliográficas}

1. Stenstedt A. A study in manic depressive psychosis: clinical, social and genetic investigations. Acta Psychiatr Scand Suppl 1952;79:1-112.

2. Perris C. A study of bipolar (manic-depressive) and unipolar recurrent depressive psychoses. I. Genetic investigation. Acta Psychiatr Scand Suppl 1966;194:15-44.

3. Angst J. On the etiology and nosology of endogenous depressive psychoses. A genetic, sociologic and clinical study. Monographien aus dem Gesamtgebiete der Neurologie und Psychiatrie 1966;112:1-118.

4. Goetzl U, Green R, Whybrow P, Jackson R. X linkage revisited. A further family study of manic-depressive illness. Arch Gen Psychiatry 1974;31:665-72.

5. Helzer JE, Winokur G. A family interview study of male manic depressives. Arch Gen Psychiatry 1974;31:73-7.

6. Gershon ES, Mark A, Cohen N, Belizon N, Baron M, Knobe KE. Transmitted factors in the morbid risk of affective disorders: a controlled study. J Psychiatr Res 1975;12:283-99.

7. Videbech T. A study of genetic factors, childhood bereavement, and premorbid personality traits in patients with anancastic endogenous depression. Acta Psychiatr Scand 1975;52:178-222.

8. James NM, Chapman CJ. A genetic study of bipolar affective disorder. Br J Psychiatry 1975;126:449-56.

9. Angst J, Frey R, Lohmeyer B, Zerbin-Rudin E. Bipolar manicdepressive psychoses: Results of a genetic investigation. Hum Genet 1980;55:237-54.

10. Tsuang MT, Winokur G, Crowe RR. Morbidity risks of schizophrenia and affective disorders among first degree relatives of patients with schizophrenia, mania, depression, and surgical conditions. Br J Psychiatry 1980;137:497-504.

11. Taylor MA, Abrams R, Hayman MA. The classification of affective disorders - a reassessment of the bipolar-unipolar dichotomy. A clinical, laboratory, and family study. J Affect Disord 1980;2:95-109.
12. Gershon ES, Hamovit J, Guroff JJ, Dibble E, Leckman JF, Sceery W et al. A family study of schizoaffective, bipolar I, bipolar II, unipolar and normal control probands. Arch Gen Psychiatry 1982;39:1157-67.

13. Baron M, Gruen R, Asnis L, Kane J. Schizoaffective illness, schizophrenia and affective disorders: morbidity risk and genetic transmission. Acta Psychiatr Scand 1982;65:253-62.

14. Fieve RR, Go R, Dunner DL, Elston R. Search for biological/genetic markers in a long-term epidemiological and morbid risk study for affective disorders. J Psychiatr Res 1984;18:425-45.

15. Coryell W, Endicott J, Andreasen N, Keller M. Bipolar I, bipolar II, and nonbipolar major deperssion among the relatives of affectively ill probands. Am J Psychiatry 1985;142:7:817-21.

16. Tsuang MT, Faraone SV, Fleming JA. Familial transmission of major affective disorders. Is there evidence supporting the distinction between unipolar and bipolar disorders. Br J Psychiatry 1985;146:26871.

17. Rice J, Reich T, Andreasen NC, Endicott J, Van Eerdewegh M, Fishman R et al. The familial transmission of bipolar illness. Arch Gen Psychiatry 1987;44:441-7.

18. Gershon ES, Berrettini W, Nurnberger JIJ et al. Genetics of affective disorders. In: Meltzer HY, editors. Psychopharmacology: The Third Generation of Progress. New York: Raven Press; 1987. p. 481-91.

19. Alda M. Bipolar disorder: from families to genes. Can J Psychiatry 1999;42:378-87.

20. Reich T, Clayton PJ, Winokur G. Family history studies: V. The genetics of mania. Am J Psychiatry 1969;125:1358-69.

21. Winokur G, Tanna VL. Possible role of X-linked dominant factor in manic depressive disease. Diseases of the Nervous System 1969;30:8994.

22. Mendlewicz J, Fleiss JL, Fieve RR. Evidence for X-linkage in the transmission of manic-depressive illness. JAMA 1972;222:1624-7. 
23. Pekkarinen $\mathrm{P}$, Terwillinger J, Bredbacka PE, Lonnqvist J, Peltonen L. Evidence of a predisposing locus to bipolar disorder on Xq24-q27.1 in an extended finnish pedigree. Genome Res 1995;5:105-15.

24. Hebebrand J. A critical appraisal of X-linked bipolar illness: evidence for the assumed mode of inheritance is lacking. Br J Psychiatry 1992;160:7-11.

25. Baron M, Risch N, Hamburger R, Mandel B, Kushner S, Newman $\mathrm{M}$ et al. Genetic linkage between X-chromosome markers and bipolar affective illness. Nature 1987;326:289-92.

26. Gershon ES, Bunney WEJ. The question of X-linkage in bipolar manic-depressive illness. J Psychiatr Res 1977;13:99-117.

27. Baron M, Hamburger R, Sandkuyl LA, Risch N, Mandel B, Endicott $\mathrm{J}$ et al. The impact of phenotypic variation on genetic analysis: application to X-linkage in manic-depressive illness. Acta Psychiatr Scand 1990;82:196-203.

28. Egeland JA, Gerhard DS, Pauls DL, Sussex JN, Kidd KK, Allen CR et al. Bipolar affective disorders linked to DNA markers on chromosome 11. Nature 1987;325:783-7.

29. Kelsoe JR, Ginns EI, Egeland JA, Gerhard DS, Goldstein AM, Bale SJ et al. Re-evaluation of the linkage relationship between chromosome $11 \mathrm{p}$ loci and the gene for bipolar affective disorder in the Old Order Amish. Nature 1989;342:238-43.

30. Berrettini WH, Ferraro TN, Goldin LR, Weeks DE, DeteraWadleigh S, Nurnberger JIJ et al. Chromosome 18 DNA markers and manic-depressive illness: evidence for a susceptibility gene. Proc Natl Acad Sci USA 1994;91:5918-21.

31. Stine OC, Xu J, Koskela R, McMahon FJ, Gschwend M, Friddle C et al. Evidence for linkage of bipolar disorder to chromosome 18 with a parent-of-origin effect. Am J Hum Genet 1995;57:1384-94.

32. Detera-Wadleigh SD, Badner JA, Berrettini WH, Yoshikawa T, Goldin LR, Turner $\mathrm{G}$ et al. A high-density genome scan detects evidence for a bipolar-disorder susceptibility locus on $13 \mathrm{q} 32$ and other potential loci on 1q32 and 18p11.2. Proc Natl Acad Sci USA 1999;96:5604-9.

33. Freimer NB, Reus VI, Escamilla MA, McInnes LA, Spesny M, Leon $\mathrm{P}$ et al. Genetic mapping using haplotype, association and linkage methods suggests a locus for severe bipolar disorder (BPI) at 18q22-q23. Nat Genet 1996;12:436-41.

34. Pauls DL, Ott J, Paul SM, Allen CR, Fann CSJ, Carulli JP et al. Linkage analyses of choromosome 18 markers do not identify a major susceptibility locus for bipolar affective disorder in the old order Amish. Am J Hum Genet 1995;57:636-43.

35. Turecki G, Grof P, Cavazzoni P, Duffy A, Grof E, Martin R et al. Lithium responsive bipolar disorder, unilineality, and chromosome 18: A linkage study. Am J Med Genet (Neuropsychiatric Genet) 1999;88:411-5.

36. Bowen T, Kirov G, Gill M, Spurlock G, Vallada H, Murray RM et al. Linkage studies of bipolar disorder with chromosome 18 markers. Am J Med Genet (Neuropsychiatric Genet) 1999;88:503-9.
37. Straub RE, Lehner T, Luo Y, Loth JE, Wei S, Sharpe L et al. A possible vulnerability locus for bipolar affective disorder on chromosome 21q22.3. Nat Genet 1994;8:291-6.

38. Detera-Wadleigh SD, Badner JA, Goldin LR, Berrettini WH, Sanders AR, Rollins DY et al. Affected-sib-pair analyses reveal support of prior evidence for a susceptibility locus for bipolar disorder, on 21q. Am J Hum Genet 1996;58:1279-85.

39. Aita VM, Liu JJ, Knowles JA, Terwilliger JD, Baltazar R, Grunn A et al. A comprehensive linkage analysis of chromosome $21 \mathrm{q} 22$ supports prior evidence for a putative bipolar affective disorder locus. Am J Hum Genet 1999;64:210-7.

40. Morissette J, Villeneuve A, Bordeleau L, Rochette D, Laberge C, Gagne B et al. Genome-wide search for linkage of bipolar affective disorders in a very large pedigree derived from a homogeneous population in Quebec points to a locus of major effect on chromosome 12q23-q24. Am J Med Genet (Neuropsychiatric Genet) 1999;88:567-87.

41. Blackwood DHR, He L, Morris SW, McLean A, Whitton C, Thomson $\mathrm{M}$ et al. A locus for bipolar affective disorder on chromosome 4p. Nat Genet 1996;12:427-30.

42. Ewald H, Degn B, Mors O, Kruse TA. Support for the possible locus on chromosome $4 \mathrm{p} 16$ for bipolar affective disorder. Mol Psychiatry 1998;3:442-8.

43. Nurnberger JI, DePaulo JR, Gershon ES, Reich T, Blehar MC, Edenberg HJ et al. Genomic survey of bipolar illness in the NIMH genetics initiative pedigrees - a preliminary report. Am J Med Genet 1997;74:227-37.

44. Ginns EI, St Jean P, Philibert RA, Galdzicka M, Damschroder W, Thiel B et al. A genome-wide search for chromosomal loci linked to mental health wellness in relatives at high risk for bipolar affective disorder among the Old Order Amish. Proc Natl Acad Sci USA 1998;95:15531-6.

45. O'Donovan MC, Guy C, Craddock N, Bowen T, McKeon P, Macedo A et al. Confirmation of association between expanded CAG/CTG repeats and both schizophrenia and bipolar disorder. Psychol Med 1996;26:1145-53.

46. Craddock N, McKeon P, Moorhead S, Guy C, Harrison D, MynettJohnson L et al. Expanded CAG/CTG repeats in bipolar disorder: no correlation with phenotypic measures of illness severity. Biol Psychiatry 1997;42:876-81.

47. Grof P, Alda M, Grof E, Zvolsky P, Walsh M. Lithium response and genetics of affective disorders. J Affect Disord 1994;32:85-95.

48. Serretti A, Franchini L, Gasperini M, Rampoldi R, Smeraldi E. Mode of inheritance in mood disorder families according to fluvoxamine response. Acta Psychiatr Scand 1998;98:443-50.

49. Kutcher S, Marton P. Affective disorders in first-degree relatives of adolescent onset bipolars, unipolars, and normal controls. J Am Acad Child Adolesc Psychiatry 1991;30:75-8.

Correspondência: Martin Alda

Department of Psychiatry - Dalhousie University Abbie J. Lane Building 5909 Jubilee Road Halifax, Nova Scotia B3H 2E2 Canada Email: malda@is.dal.ca 